\title{
Application of the proximity force approximation to gravitational and Yukawa-type forces
}

\author{
R. S. Decca, ${ }^{1}$ E. Fischbach ${ }^{2}$ G. L. Klimchitskaya,${ }^{3}$ \\ D. E. Krause ${ }^{4,2}$ D. López ${ }^{5}$ and V. M. Mostepanenko ${ }^{6}$ \\ ${ }^{1}$ Department of Physics, Indiana University-Purdue \\ University Indianapolis, Indianapolis, Indiana 46202, USA \\ ${ }^{2}$ Department of Physics, Purdue University, \\ West Lafayette, Indiana 4790\%, USA \\ ${ }^{3}$ North-West Technical University, Millionnaya St. 5, St.Petersburg, 191065, Russia \\ ${ }^{4}$ Physics Department, Wabash College, \\ Crawfordsville, Indiana 47933, USA \\ ${ }^{5}$ Center for Nanoscale Materials, Argonne \\ National Laboratory, Argonne, Illinois 60439, USA \\ ${ }^{6}$ Noncommercial Partnership "Scientific Instruments", \\ Tverskaya St. 11, Moscow, 103905, Russia
}

\begin{abstract}
We apply the proximity force approximation, which is widely used for the calculation of the Casimir force between bodies with nonplanar boundary surfaces, to gravitational and Yukawa-type interactions. It is shown that for the gravitational force in a sphere-plate configuration the general formulation of the proximity force approximation is well applicable. For a Yukawa-type interaction we demonstrate the validity of both the general formulation of the proximity force approximation, and a simple mapping between the sphere-plate and plate-plate configurations. The claims to the contrary in some recent literature are thus incorrect. Our results justify the constraints on the parameters of non-Newtonian gravity previously obtained from the indirect dynamic measurements of the Casimir pressure.
\end{abstract}

PACS numbers: 04.50.-h, 14.80.-j 


\section{INTRODUCTION}

During the last few years, much attention has been devoted to experimental tests of Newton's gravitational law at short separations, and to the search for possible corrections to it (see, e.g., monograph [1] and review [2]). These corrections arise from the exchange of massless or light elementary particles predicted in many models containing a spontaneously or weakly dynamically broken symmetry. For example, the exchange of light bosons, such as scalar axions, graviphotons, dilatons and moduli (see, e.g., Refs. [3, 4]) generates a Yukawatype correction to Newtonian gravity. An almost identical correction is predicted in extradimensional physics with compact extra dimensions and low-energy compactification scale of order $1 \mathrm{TeV}[\underline{5}, \underline{6}, \mathbf{7}, \underline{8}]$.

Constraints on corrections to Newton's gravitational law at short separations can be obtained from precise force measurements between macrobodies. At separations greater than $10^{-5} \mathrm{~m}$ the dominant force between electrically neutral test bodies is gravity, and recent new strong constraints on the Yukawa-type interaction in sub-millimeter range have been

obtained in gravity experiments $[9,10,11,12,13,14,15]$. In the sub-micrometer range the dominant forces are the Casimir and van der Waals forces [16]. Here the strongest constraints on Yukawa-type corrections to Newtonian gravity were obtained from measurements of the Casimir force [17, 18, 19, 20, 21, 22, 23, 24, 25].

All precise measurements of the Casimir force which were used to obtain constraints on non-Newtonian gravity were performed in a sphere-plate configuration, rather than in a plate-plate configuration. The reason is that it is hard to ensure that two plates are parallel at separations $a$ below $a=1 \mu \mathrm{m}$ with sufficient precision. The Casimir force in a sphere-plate configuration $F_{C}(a)$ can be obtained from the Casimir energy per unit area in a configuration of two parallel plates, $E_{C}(a)$, by means of

$$
F_{C}(a)=2 \pi R E_{C}(a)
$$

where $R$ is the sphere radius. This equation represents the application of the general approximate method, the proximity force approximation (PFA) [26], for this geometry. As was demonstrated recently both theoretically [27, 28, 29] and experimentally [30], the PFA provides a very precise determination of the Casimir force under the condition that $a \ll R$. The relative corrections to the value of the force calculated using Eq. (1) were shown to be less than $a / R$, and for typical $a$ and $R$ used in experiments this is only $0.1 \%$ of the Casimir 
force. In the dynamic measurement regime, the PFA gives the possibility to determine the Casimir pressure between two parallel plates from experimental data for the gradient of the Casimir force in a sphere-plate configuration.

It has been claimed recently [31] that the PFA "does not hold for forces of volumetric character such as the gravitational force or its hypothetical short-range relatives". On this basis the limits on the Yukawa force obtained from the dynamic measurements of the Casimir pressure between two parallel plates using a sphere-plate configuration, as in [21] and some other experiments, were called "invalid". Below we apply the PFA to both gravitational and Yukawa-type forces and demonstrate that the results that are obtained agree nicely with the results of direct calculations that do not use the PFA. Statements to the contrary made in Ref. [31] with respect to gravitational force are explained by a confusion with different formulations of the PFA. With respect to the Yukawa-type interaction these statements are shown to be incorrect.

The paper is organized as follows. In Sec. II we present different formulations of the proximity force approximation and apply them to the gravitational interaction. Section III demonstrates that the proximity force approximation is readily applicable to Yukawa-type hypothetical forces. Section IV establishes the applicability of the PFA to Yukawa forces in dynamic experiments constituting an indirect measurement of the Casimir pressure between two parallel plates using the sphere-plate configuration. In Sec. V we present our conclusions and discussion.

\section{THE PROXIMITY FORCE APPROXIMATION AND NEWTONIAN GRAV- ITY}

In the most general formulation of the PFA [26], the $z$-component of the force acting between two arbitrarily shaped bodies $V_{1}$ and $V_{2}$ can be approximately represented as a sum of forces between plane parallel surface elements $d x d y$

$$
F_{z}(a)=\iint_{\sigma} d x d y P(x, y, z(x, y))
$$

Here, $P(x, y, z(x, y))$ is the known pressure for a configuration of two parallel plates, $z(x, y)=z_{2}(x, y)-z_{1}(x, y)$, where $z_{2}(x, y)>z_{1}(x, y)$ are the surfaces of the bodies arranged against each other in an appropriate coordinate system, $\sigma$ is the part of the $(x, y)$-plane, 
where both surfaces are defined, and $a$ is the smallest value of $z(x, y)$.

In Ref. [32] Eq. (2) was applied to the case of smooth interacting surfaces having a single point $x=y=0$ where the width of the gap $z(x, y)$ reaches a minimum. It was also assumed that the characteristic size of the upper body in the $z$-direction extends beyond the range of $z$ where the pressure $P(x, y, z(x, y))$ drops to zero, so that the integral becomes independent of its upper limit. Under these conditions Eq. (21) yielded [32]

$$
F_{z}(a)=2 \pi \bar{R} E(a)
$$

where $E(a)$ is the energy per unit area in the configuration of two parallel plates of infinite area, interacting via the same force as the bodies $V_{1}$ and $V_{2}, \bar{R}=\sqrt{R_{x} R_{y}}$ and $R_{x}, R_{y}$ are the principal radii of curvature at the point $(0,0)$. For a sphere at short separations above a plate $(a \ll R)$ interacting via the Casimir force, Eq. (3) transforms into Eq. (1).

We next check the applicability of the PFA for the calculation of the gravitational force acting between a large plane plate of mass density $\rho_{1}$ and thickness $D_{1}$ and a spherical ball (sphere) of radius $R$ and density $\rho_{2}$ spaced at a height $a$ above this plate (see Fig. 1). The exact calculation of the gravitational force in this configuration is straightforward. For this purpose we first consider the point-like mass $m_{2}$ at a point $\boldsymbol{r}_{2}$ belonging to the sphere interacting via Newton's force with any point-like mass $m_{1}$ at a point $\boldsymbol{r}_{1}$ belonging to the plate

$$
\boldsymbol{F}=-G m_{1} m_{2} \frac{\boldsymbol{r}_{2}-\boldsymbol{r}_{1}}{\left|\boldsymbol{r}_{2}-\boldsymbol{r}_{1}\right|^{3}},
$$

where $G$ is the gravitational constant. The $z$-component of the force acting between the mass $m_{2}$ and the plate is obtained by integration of (41) over the plate volume

$$
\begin{aligned}
F_{z, \mathrm{gr}}^{m_{2}} & =-2 \pi G m_{2} \rho_{1} \int_{-D_{1}}^{0} d z_{1} \int_{0}^{\infty} r d r \frac{z_{2}-z_{1}}{\left[r^{2}+\left(z_{2}-z_{1}\right)^{2}\right]^{3 / 2}} \\
& =-2 \pi G m_{2} \rho_{1} D_{1},
\end{aligned}
$$

where the $(x, y)$-plane coincides with the upper boundary surface of the plate, and $r^{2}=$ $x^{2}+y^{2}$.

Note that the force (5) does not depend on $z_{2}$. Because of this the integration over the volume of the sphere reduces to the multiplication by its volume. Thus the gravitational force between a sphere and a plate is given by

$$
F_{z, \mathrm{gr}}^{s p}=-\frac{8 \pi^{2}}{3} G \rho_{1} \rho_{2} D_{1} R^{3}
$$


From Eq. (5) it is also easy to obtain the exact expression for the gravitational pressure between the two parallel plates of infinite area, lower and upper, with parameters $\rho_{1}, D_{1}$ and $\rho_{2}, D_{2}$, respectively

$$
P_{\mathrm{gr}}=-2 \pi G \rho_{1} \rho_{2} D_{1} D_{2} .
$$

We emphasize that this pressure does not depend on the separation between the plates.

Next we apply the most general formulation (2) of the PFA to calculate the gravitational force in the sphere-plate configuration. The pressure $P(x, y, z(x, y))$ in Eq. (2) is given by Eq. (77) where in accordance with Fig. $1 D_{2}$ should be replaced by

$$
D_{2}(x, y)=2 \sqrt{R^{2}-x^{2}-y^{2}}=2 \sqrt{R^{2}-r^{2}} .
$$

Substituting Eqs. (7) and (8) in Eq. (2), we obtain the PFA result for the gravitational force acting between a sphere and a plate

$$
\begin{aligned}
F_{z, \mathrm{gr}}^{s p} & =-8 \pi^{2} G \rho_{1} \rho_{2} D_{1} \int_{0}^{R} r d r \sqrt{R^{2}-r^{2}} \\
& =-\frac{8 \pi^{2}}{3} G \rho_{1} \rho_{2} D_{1} R^{3},
\end{aligned}
$$

which is identical to the exact result (6). The fact that in a sphere-plate configuration the PFA leads to the exact result is explained by the additivity of the gravitational interaction. It is important to keep in mind, however, that Eq. (9) cannot be obtained using the second, simplified, formulation of the PFA given by Eq. (3). The reason is that $P_{\mathrm{gr}}$ in Eq. (77) does not drop to zero within the volume of a sphere which makes Eq. (3) inapplicable.

\section{THE PROXIMITY FORCE APPROXIMATION AND YUKAWA-TYPE IN- TERACTION}

We next consider a Yukawa-type interaction potential between the point-like material particles

$$
V_{\mathrm{Yu}}\left(\left|\boldsymbol{r}_{2}-\boldsymbol{r}_{1}\right|\right)=-G m_{1} m_{2} \alpha \frac{1}{\left|\boldsymbol{r}_{2}-\boldsymbol{r}_{1}\right|} \mathrm{e}^{-\left|\boldsymbol{r}_{2}-\boldsymbol{r}_{1}\right| / \lambda},
$$

where $\alpha$ is the dimensionless interaction strength relative to gravity, and $\lambda$ is the interaction range, and we calculate the respective force acting between a sphere and a plate. Keeping in mind that this interaction is very weak, and is not of electromagnetic origin, it can be considered as additive to a very high degree of accuracy. We begin with the calculation of 
the Yukawa force acting between a sphere and a plate by directly performing the additive summation of potentials (10).

By considering a particle $m_{2}$ at a height $z$ above a large plate with thickness $D_{1}$ and density $\rho_{1}$, we obtain the Yukawa energy of their interaction by integration of Eq. (10) over the volume of the plate

$$
E_{\mathrm{Yu}}^{m_{2}}(z)=-2 \pi G m_{2} \rho_{1} \alpha \lambda^{2} \mathrm{e}^{-z / \lambda}\left(1-\mathrm{e}^{-D_{1} / \lambda}\right)
$$

Calculating the negative derivative of this expression with respect to $z$, one finds the $z$ component of the Yukawa force acting between a particle and a plate

$$
F_{z, \mathrm{Yu}}^{m_{2}}(z)=-2 \pi G m_{2} \rho_{1} \alpha \lambda \mathrm{e}^{-z / \lambda}\left(1-\mathrm{e}^{-D_{1} / \lambda}\right)
$$

Integrating this equation over the volume of the sphere (see Fig. 1), one obtains the desired result for the Yukawa force in a sphere-plate configuration

$$
\begin{aligned}
& F_{z, \mathrm{Yu}}^{s p}(a)=-2 \pi^{2} G \rho_{1} \rho_{2} \alpha \lambda\left(1-\mathrm{e}^{-D_{1} / \lambda}\right) \\
& \times \int_{a}^{2 R+a} d z\left[R^{2}-(R+a-z)^{2}\right] \mathrm{e}^{-z / \lambda} \\
&=-4 \pi^{2} G \rho_{1} \rho_{2} \alpha \lambda^{3}\left(1-\mathrm{e}^{-D_{1} / \lambda}\right) R \mathrm{e}^{-a / \lambda} \\
& \times\left(1-\frac{\lambda}{R}+\mathrm{e}^{-2 R / \lambda}+\frac{\lambda}{R} \mathrm{e}^{-2 R / \lambda}\right) .
\end{aligned}
$$

From the integration of Eq. (12) over the volume of the upper plate one can also obtain the expression for the Yukawa pressure between the two parallel plates of infinite area spaced at a separation $a$ with the parameters $\rho_{1}, D_{1}$ (the lower plate) and $\rho_{2}, D_{2}$ (the upper plate)

$$
P_{\mathrm{Yu}}(a)=-2 \pi G \rho_{1} \rho_{2} \alpha \lambda^{2} \mathrm{e}^{-a / \lambda}\left(1-\mathrm{e}^{-D_{1} / \lambda}\right)\left(1-\mathrm{e}^{-D_{2} / \lambda}\right)
$$

In a similar way, integrating Eq. (11) over the volume of an upper plate we arrive at the Yukawa energy per unit area in the configuration of two parallel plates

$$
E_{\mathrm{Yu}}(a)=-2 \pi G \rho_{1} \rho_{2} \alpha \lambda^{3} \mathrm{e}^{-a / \lambda}\left(1-\mathrm{e}^{-D_{1} / \lambda}\right)\left(1-\mathrm{e}^{-D_{2} / \lambda}\right)
$$

We now turn to the application of the PFA to the Yukawa interaction starting from the most general formulation of PFA given by Eq. (2). For this purpose we replace $a$ in Eq. (14) by $R+a-\sqrt{R^{2}-r^{2}}$ (which is the separation between the plane plates in accordance with Fig. 1) and choose $D_{2}$ as in Eq. (8). Substituting the resulting expression 
for $P_{\mathrm{Yu}}(x, y, z(x, y))$ in Eq. (2) and performing the integration one finds the PFA result for the Yukawa interaction in a sphere-plate configuration

$$
\begin{aligned}
F_{z, \mathrm{Yu}}^{s p}(a)=- & 4 \pi^{2} G \rho_{1} \rho_{2} \alpha \lambda^{2}\left(1-\mathrm{e}^{-D_{1} / \lambda}\right) \\
& \times \int_{0}^{R} r d r \mathrm{e}^{-\left(R+a-\sqrt{R^{2}-r^{2}}\right) / \lambda}\left(1-\mathrm{e}^{-2 \sqrt{R^{2}-r^{2}} / \lambda}\right) \\
=- & 4 \pi^{2} G \rho_{1} \rho_{2} \alpha \lambda^{3}\left(1-\mathrm{e}^{-D_{1} / \lambda}\right) R \mathrm{e}^{-a / \lambda} \\
& \times\left(1-\frac{\lambda}{R}+\mathrm{e}^{-2 R / \lambda}+\frac{\lambda}{R} \mathrm{e}^{-2 R / \lambda}\right) .
\end{aligned}
$$

It is seen that Eq. (16) is identical to Eq. (13). Thus, for the Yukawa interaction the most general formulation of the PFA leads to exactly the same result as the additive summation of potentials (10).

Now we consider a more subtle situation with the simplified formulation of the PFA given by Eq. (3). From the comparison of Eqs. (15) and (16) it is seen that $F_{z, \mathrm{Yu}}^{s p}(a)$ is not obtained as $E_{\mathrm{Yu}}(a)$ times $2 \pi R$, as is required by Eq. (3). However, in the case of a sufficiently thick upper plate $\left(D_{2} \gg \lambda\right)$ and large sphere $(R \gg \lambda)$ one arrives from Eqs. (15), (16) at

$$
\begin{aligned}
F_{z, \mathrm{Yu}}^{s p}(a) & =-4 \pi^{2} G \rho_{1} \rho_{2} \alpha \lambda^{3}\left(1-\mathrm{e}^{-D_{1} / \lambda}\right) R \mathrm{e}^{-a / \lambda} \\
& =2 \pi R E_{\mathrm{Yu}}(a)
\end{aligned}
$$

in accordance with the simplified formulation of the PFA in Eq. (3)). Thus, the simplified formulation of the PFA works well for a Yukawa-type interaction providing the mapping between the configurations of a large sphere $(R \gg \lambda)$ above a plate and the same plate parallel to another (thick) plate.

\section{LAYERED STRUCTURES USED IN DYNAMIC EXPERIMENTS}

We next consider the application of the above results to the configuration of experiments in Refs. [20, 21, 22, 23]. In these experiments, the Casimir pressure between two parallel plates was measured indirectly in the dynamic mode using experimental data for the gradient

of the Casimir force, acting between a sphere and a plate, and the PFA. The extent of agreement of the resulting data for the Casimir pressure with theory was used to obtain constraints on a Yukawa-type hypothetical interaction. In so doing the expression for the Yukawa pressure in the configuration of two parallel plates has been used. To justify this 
calculation procedure we require that the PFA provide a similar mapping between the sphereplate and plate-plate configurations for both Casimir and Yukawa-type forces. The latter was questioned in Ref. [31], but shown to be correct in Eq. (17) for the case of a homogeneous plate $\left(D_{2} \gg \lambda\right)$ and sphere $(R \gg \lambda)$ with densities $\rho_{1}$ and $\rho_{2}$, respectively.

In the experiments of Refs. [20, 21, 22, 23], however, the plate and the sphere were not homogeneous but covered with thin metallic layers with thicknesses $\Delta_{1}^{\prime}, \Delta_{1}^{\prime \prime}$ and densities $\rho_{1}^{\prime}, \rho_{1}^{\prime \prime}$ (for the plate) and thicknesses $\Delta_{2}^{\prime}, \Delta_{2}^{\prime \prime}$ and densities $\rho_{2}^{\prime}, \rho_{2}^{\prime \prime}$ (for the sphere). Below we check that the mapping provided by the PFA for the configurations of sphere-plate and plate-plate in the case of Yukawa interaction with $\lambda \ll R$ is preserved in the presence of these layers. We first note that the plate thickness $\left(D_{1}=3.5 \mu \mathrm{m}\right)$ is much larger than the typical interaction range of the Yukawa interaction under consideration $(\lambda=0.1 \mu \mathrm{m})$. Because of this, the term $\exp \left(-D_{1} / \lambda\right)$ in Eq. (17) is negligibly small as compared with unity and can be omitted. The energy per unit area in a plate-plate configuration and the Yukawa force in a sphere-plate configuration can then be simplified to the form

$$
\begin{aligned}
& E_{\mathrm{Yu}}(a)=-2 \pi G \rho_{1} \rho_{2} \alpha \lambda^{3} \mathrm{e}^{-a / \lambda}, \\
& F_{z, \mathrm{Yu}}^{s p}(a)=-4 \pi^{2} G \rho_{1} \rho_{2} \alpha \lambda^{3} R \mathrm{e}^{-a / \lambda} .
\end{aligned}
$$

Using Eq. (18) it is a simple matter to take into consideration thin layers covering the test bodies in the experiments of Refs. [20, 21, 22, 23]. As an example, let us consider the configuration of two homogeneous plates with the densities $\rho_{1}$ (the lower plate) and $\rho_{2}$ (the upper plate) at a separation $a+\Delta_{1}^{\prime}$, and assume that the lower plate is covered with an additional layer of thickness $\Delta_{1}^{\prime}$ and density $\rho_{1}^{\prime}$. We then apply the first equality in Eq. (18) to two thick plates with densities $\rho_{1}^{\prime}$ and $\rho_{2}$ at a separation $a$ and subtract from the resulting expression the Yukawa energy per unit area between two thick plates with densities $\rho_{1}^{\prime}$ and $\rho_{2}$ at a separation $a+\Delta_{1}^{\prime}$. This gives us the Yukawa energy per unit area between the thin layer of thickness $\Delta_{1}^{\prime}$ and density $\rho_{1}^{\prime}$ spaced $a$ apart from the thick upper plate of density $\rho_{2}$

$$
E_{\mathrm{Yu}}^{\Delta_{1}^{\prime}}(a)=-2 \pi G \rho_{1}^{\prime} \rho_{2} \alpha \lambda^{3} \mathrm{e}^{-a / \lambda}\left(1-\mathrm{e}^{-\Delta_{1}^{\prime} / \lambda}\right)
$$

We next combine Eq. (19) with the first equality in Eq. (18) written for the Yukawa energy between two thick plates of densities $\rho_{1}$ and $\rho_{2}$ at a separation $a+\Delta_{1}^{\prime}$ and obtain the desired result for the two plates one of which is covered with a thin layer

$$
E_{\mathrm{Yu}}(a)=-2 \pi G \rho_{2} \alpha \lambda^{3} \mathrm{e}^{-a / \lambda}\left[\rho_{1}^{\prime}-\left(\rho_{1}^{\prime}-\rho_{1}\right) \mathrm{e}^{-\Delta_{1}^{\prime} / \lambda}\right] .
$$


By repeating the same procedure for each of the two thin layers covering the lower and upper plate one arrives at

$$
\begin{aligned}
& E_{\mathrm{Yu}}^{\Delta}(a)=-2 \pi G \alpha \lambda^{3} \mathrm{e}^{-a / \lambda} \\
& \quad \times\left[\rho_{1}^{\prime \prime}-\left(\rho_{1}^{\prime \prime}-\rho_{1}^{\prime}\right) \mathrm{e}^{-\Delta_{1}^{\prime \prime} / \lambda}-\left(\rho_{1}^{\prime}-\rho_{1}\right) \mathrm{e}^{-\left(\Delta_{1}^{\prime \prime}+\Delta_{1}^{\prime}\right) / \lambda}\right] \\
& \times\left[\rho_{2}^{\prime \prime}-\left(\rho_{2}^{\prime \prime}-\rho_{2}^{\prime}\right) \mathrm{e}^{-\Delta_{2}^{\prime \prime} / \lambda}-\left(\rho_{2}^{\prime}-\rho_{2}\right) \mathrm{e}^{-\left(\Delta_{2}^{\prime \prime}+\Delta_{2}^{\prime}\right) / \lambda}\right] .
\end{aligned}
$$

The vertical component of the Yukawa force given by the second equality in Eq. (18) can also be easily rewritten for the case when both the sphere and the plate are covered with two thin layers

$$
\begin{aligned}
& F_{z, \mathrm{Yu}}^{s p, \Delta}(a)=-4 \pi^{2} G \alpha \lambda^{3} \mathrm{e}^{-a / \lambda} \\
& \quad \times\left[\rho_{1}^{\prime \prime}-\left(\rho_{1}^{\prime \prime}-\rho_{1}^{\prime}\right) \mathrm{e}^{-\Delta_{1}^{\prime \prime} / \lambda}-\left(\rho_{1}^{\prime}-\rho_{1}\right) \mathrm{e}^{-\left(\Delta_{1}^{\prime \prime}+\Delta_{1}^{\prime}\right) / \lambda}\right] \\
& \quad \times\left[R \rho_{2}^{\prime \prime}-\left(R-\Delta_{2}^{\prime \prime}\right)\left(\rho_{2}^{\prime \prime}-\rho_{2}^{\prime}\right) \mathrm{e}^{-\Delta_{2}^{\prime \prime} / \lambda}-\left(R-\Delta_{2}^{\prime \prime}-\Delta_{2}^{\prime}\right)\left(\rho_{2}^{\prime}-\rho_{2}\right) \mathrm{e}^{-\left(\Delta_{2}^{\prime \prime}+\Delta_{2}^{\prime}\right) / \lambda}\right] .
\end{aligned}
$$

From the comparison of Eqs. (21) and (22) it may seem that the equation

$$
F_{z, \mathrm{Yu}}^{s p, \Delta}(a)=2 \pi R E_{\mathrm{Yu}}^{\Delta}(a)
$$

providing a mapping between the configurations of sphere-plate and plate-plate does not hold for a Yukawa-type interaction. One should, however, take into account the values of layer thicknesses used in the experiments [20, 21, 22, 23], as compared to sphere radii. Thus, the thicknesses of the first covering layers vary from $\Delta_{1}^{\prime}=\Delta_{2}^{\prime}=1 \mathrm{~nm}[20]$ to $\Delta_{1}^{\prime}=\Delta_{2}^{\prime}=10 \mathrm{~nm}$ $[21,22,23]$. The thicknesses of the external covering layers are equal to $\Delta_{1}^{\prime \prime}=200 \mathrm{~nm}$, $\Delta_{2}^{\prime \prime}=203 \mathrm{~nm}[20], \Delta_{1}^{\prime \prime}=150 \mathrm{~nm}, \Delta_{2}^{\prime \prime}=200 \mathrm{~nm}[21,22]$, and $\Delta_{1}^{\prime \prime}=210 \mathrm{~nm}, \Delta_{2}^{\prime \prime}=180 \mathrm{~nm}$ [23]. The spheres used in these experiments have the radii $R=294.3 \mu \mathrm{m} \mathrm{[20],} 148.7 \mu \mathrm{m}$ [21, 22], and $151 \mu \mathrm{m}[23]$. If we rewrite Eq. (22) in the form

$$
\begin{aligned}
& F_{z, \mathrm{Yu}}^{s p, \Delta}(a)=-4 \pi^{2} G \alpha \lambda^{3} \mathrm{e}^{-a / \lambda} R \\
& \times\left[\rho_{1}^{\prime \prime}-\left(\rho_{1}^{\prime \prime}-\rho_{1}^{\prime}\right) \mathrm{e}^{-\Delta_{1}^{\prime \prime} / \lambda}-\left(\rho_{1}^{\prime}-\rho_{1}\right) \mathrm{e}^{-\left(\Delta_{1}^{\prime \prime}+\Delta_{1}^{\prime}\right) / \lambda}\right] \\
& \quad \times\left[\rho_{2}^{\prime \prime}-\left(\rho_{2}^{\prime \prime}-\rho_{2}^{\prime}\right) \mathrm{e}^{-\Delta_{2}^{\prime \prime} / \lambda}-\left(\rho_{2}^{\prime}-\rho_{2}\right) \mathrm{e}^{-\left(\Delta_{2}^{\prime \prime}+\Delta_{2}^{\prime}\right) / \lambda}\right. \\
& \left.\quad+\frac{\Delta_{2}^{\prime \prime}}{R}\left(\rho_{2}^{\prime \prime}-\rho_{2}^{\prime}\right) \mathrm{e}^{-\Delta_{2}^{\prime \prime} / \lambda}+\frac{\Delta_{2}^{\prime \prime}+\Delta_{2}^{\prime}}{R}\left(\rho_{2}^{\prime}-\rho_{2}\right) \mathrm{e}^{-\left(\Delta_{2}^{\prime \prime}+\Delta_{2}^{\prime}\right) / \lambda}\right],
\end{aligned}
$$

it is easily seen that in all experiments under consideration [20, 21, 22, 23] the last two terms on the right-hand side of Eq. (24) are negligibly small, and can be discarded due to 
the inequalities

$$
\frac{\Delta_{2}^{\prime \prime}}{R} \ll 1, \quad \frac{\Delta_{2}^{\prime \prime}+\Delta_{2}^{\prime}}{R} \ll 1
$$

In so doing one neglects quantities $\approx 0.7 \times 10^{-3}$ [20], $1.4 \times 10^{-3}$ [21, 22], and $1.2 \times 10^{-3}$ [23] as compared with unity. This confirms the validity of Eq. (23) in the configurations of experiments [20, 21, 22, 23] with a very high precision (an error of about $0.1 \%$ ). This is the same level of precision with which the PFA describes the Casimir force in a sphere-plate configuration (see Sec. I).

\section{CONCLUSIONS AND DISCUSSION}

In the foregoing we have applied the proximity force approximation to gravitational and Yukawa forces. This approximate method is widely used to calculate the Casimir force in configurations with curved boundaries, but was not considered up to now in application to gravitation or Yukawa-type interactions. This allowed some doubts to be raised [31] concerning the suitability of the PFA to slowly decreasing forces of a volumetric character, and to thus question the validity of constraints obtained on such forces from dynamic measurements of the Casimir force [20, 21, 22, 23].

We have shown that care is required in the application of the PFA to gravitational forces. Thus the most general formulation of the PFA, Eq. (2), is quite applicable for the calculation of the gravitational force in a sphere-plate configuration while the simplified formulation, Eq. (3), is not. This situation is not unique. For instance, the simplified formulation is not applicable for the calculation of the Casimir force in a configuration of a thin transparent dielectric lens above a plate. At the same time, the most general formulation of the PFA works well in this case. Another example is the calculation of the electrostatic force acting between a plane and a sphere with local deviations from perfect spherical shape. Here again the most general formulation of the PFA works well [33], whereas the simplified formulation fails to provide a correct theoretical description of the electric force. By extension the PFA will also apply to inverse power laws $\sim 1 / r^{n}(n>2)$.

For Yukawa-type forces we have demonstrated the applicability of both formulations of the PFA in a sphere-plate configuration under the condition that the interaction range $\lambda$ is much smaller than the sphere radius. We have also shown that the PFA remains valid in the experimental configurations of experiments on the indirect dynamic measurement 
of the Casimir pressure between two parallel plates in the configuration of a sphere and a plate covered with additional thin layers. This allows the mapping of Yukawa interactions in a sphere-plate and a plate-plate configurations and confirms the validity of constraints on non-Newtonian gravity obtained in Refs. [20, 21, 22, 23].

\section{Acknowledgments}

R.S.D. acknowledges NSF support through Grants No. CCF-0508239 and PHY-0701636, and from the Nanoscale Imaging Center at IUPUI. E.F. was supported in part by DOE under Grant No. DE-76ER071428. G.L.K. and V.M.M. were partially supported by Deutsche Forschungsgemeinschaft, Grant No. 436 RUS 113/789/0-4 and by Purdue University.

[1] E. Fischbach and C. L. Talmadge, The Search for Non-Newtonian Gravity (Springer, New York, 1999).

[2] E. G. Adelberger, B. R. Heckel, and A. E. Nelson, Ann. Rev. Nucl. Part. Sci. 53, 77 (2003).

[3] Y. Fujii, Int. J. Mod. Phys. A 6, 3505 (1991).

[4] S. Dimopoulos and G. F. Giudice, Phys. Lett. B 379, 105 (1996).

[5] I. Antoniadis, N. Arkani-Hamed, S. Dimopoulos, and G. Dvali, Phys. Lett. B 436, 257 (1998).

[6] N. Arkani-Hamed, S. Dimopoulos, and G. Dvali, Phys. Lett. B 429, 263 (1998); Phys. Rev. D 59, 086004 (1999).

[7] E. G. Floratos and G. K. Leontaris, Phys. Lett. B 465, 95 (1999).

[8] A. Kehagias and K. Sfetsos, Phys. Lett. B 472, 39 (2000).

[9] J. C. Long, H. W. Chan, A. B. Churnside, E. A. Gulbis, M. C. M. Varney, and J. C. Price, Nature 421, 922 (2003).

[10] J. Chiaverini, S. J. Smullin, A. A. Geraci, D. M. Weld, and A. Kapitulnik, Phys. Rev. Lett. 90, 151101 (2003).

[11] S. J. Smullin, A. A. Geraci, D. M. Weld, J. Chiaverini, S. Holmes, and A. Kapitulnik, Phys. Rev. D 72, 122001 (2005).

[12] D. J. Kapner, T. S. Cook, E. G. Adelberger, J. H. Gundlach, B. R. Heckel, C. D. Hoyle, and H. E. Swanson, Phys. Rev. Lett. 98, 021101 (2007). 
[13] E. G. Adelberger, B. R. Heckel, S. Hoedl, C. D. Hoyle, D. J. Kapner, and A. Upadhye, Phys. Rev. Lett. 98, 131104 (2007).

[14] S. Schlamminger, K.-Y. Choi, T. A. Wagner, J. H. Gundlach, and E. G. Adelberger, Phys. Rev. Lett. 100, 041101 (2008).

[15] A. A. Geraci, S. J. Smullin, D. M. Weld, J. Chiaverini, and A. Kapitulnik, Phys. Rev. D 78, $022002(2008)$.

[16] M. Bordag, U. Mohideen, and V. M. Mostepanenko, Phys. Rep. 353, 1 (2001).

[17] M. Bordag, B. Geyer, G. L. Klimchitskaya, and V. M. Mostepanenko, Phys. Rev. D 58, 075003 (1998); 60, 055004 (1999); 62, 011701(R) (2000).

[18] V. M. Mostepanenko and M. Novello, Phys. Rev. D 63, 115003 (2001).

[19] E. Fischbach, D. E. Krause, V. M. Mostepanenko, and M. Novello, Phys. Rev. D 64, 075010 (2001).

[20] R. S. Decca, E. Fischbach, G. L. Klimchitskaya, D. E. Krause, D. López, and V. M. Mostepanenko, Phys. Rev. D 68, 116003 (2003).

[21] R. S. Decca, D. López, E. Fischbach, G. L. Klimchitskaya, D. E. Krause, and V. M. Mostepanenko, Ann. Phys. (N.Y.) 318, 37 (2005).

[22] G. L. Klimchitskaya, R. S. Decca, , E. Fischbach, D. E. Krause, D. López, and V. M. Mostepanenko, Int. J. Mod. Phys. A 20, 2205 (2005).

[23] R. S. Decca, D. López, E. Fischbach, G. L. Klimchitskaya, D. E. Krause, and V. M. Mostepanenko, Phys. Rev. D 75, 077101 (2007); Eur. Phys. J. C 51, 963 (2007).

[24] Ph. Brax, C. van de Bruck, A.-C. Davis, D. F. Mota, and D. Shaw, Phys. Rev. D 76, 124034 (2007).

[25] V. M. Mostepanenko, R. S. Decca, E. Fischbach, G. L. Klimchitskaya, D. E. Krause, and D. López, J. Phys. A: Math. Theor. 41, 164054 (2008).

[26] B. V. Derjaguin, Kolloid. Z. 69, 155 (1934).

[27] H. Gies and K. Klingmüller, Phys. Rev. Lett. 96, 220401 (2006).

[28] M. Bordag and V. Nikolaev, J. Phys. A: Math. Theor. 41, 164002 (2008).

[29] A. Canaguier-Durand, P. A. Maia Neto, I. Cavero-Pelaez, A. Lambrecht, and S. Reynaud, arXiv:0901.2647v1.

[30] D. E. Krause, R. S. Decca, D. López, and E. Fischbach, Phys. Rev. Lett. 98, 050403 (2007).

[31] W. J. Kim, M. Brown-Hayes, D. A. R. Dalvit, J. H. Brownell, and R. Onofrio, Phys. Rev. A 
79, 026102 (2009).

[32] J. Blocki, J. Randrup, W. J. Swiatecki, and C. F. Tsang, Ann. Phys. (N.Y.) 105, 427 (1997).

[33] R. S. Decca, E. Fischbach, G. L. Klimchitskaya, D. E. Krause, D. López, U. Mohideen, and V. M. Mostepanenko, Phys. Rev. A 79, 026101 (2009).

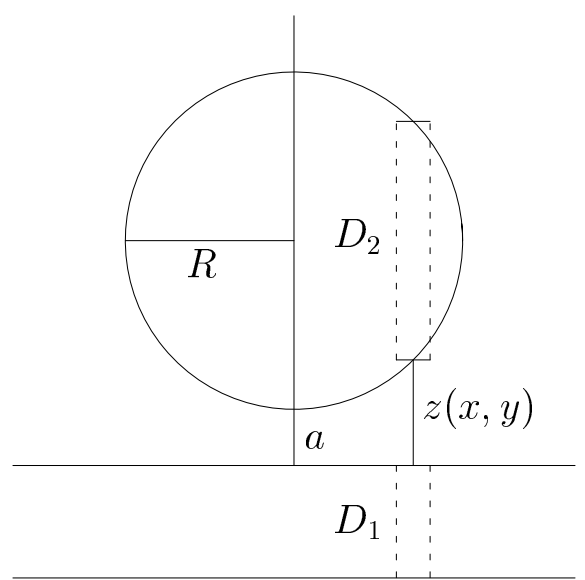

FIG. 1: Configuration of a sphere of radius $R$ spaced at the height $a$ above a plate of thickness $D_{1}$ (see text for further discussion). 